\title{
Ferramenta TIME para avaliação de feridas: concordância interobservador
}

\section{TIME wound assessment tool: interobserver agreement}

\author{
TIME para evaluación de heridas: acuerdo de interobserver
}

\begin{abstract}
Nazareno Ferreira Lopes Coutinho Júnior 1,*, Sandra Marina Gonçalves Bezerra ${ }^{1}$, Nayra Ferreira Lima Castelo Branco', Marianne Rocha Duarte de Carvalho ${ }^{1}$, Kerson Rocha Júnior ${ }^{1}$, Luís Felipe Oliveira Ferreira², Elyrose Sousa Brito Rocha ${ }^{1}$
\end{abstract}

ORCID IDs

Coutinho Júnior NFL (D) https://orcid.org/0000-0003-2110-3642

Bezerra SMG (D) https://orcid.org/0000-0003-3890-5887

Branco NFLC (D) http://orcid.org/0000-0002-2569-7647

Carvalho MRD (D) https://orcid.org/0000-0001-8479-7376

Rocha Júnior K (D) https://orcid.org/0000-0003-0204-2784

Ferreira LFO (D) https://orcid.org/0000-0002-2982-894X

Rocha ESB (D) https://orcid.org/0000-0002-7722-6564

\begin{abstract}
COMO CITAR
Coutinho Júnior NFL; Bezerra SMG; Branco NFLC; Carvalho MRD; Rocha Júnior K; Ferreira LFO; Rocha ESB. Ferramenta TIME para avaliação de feridas: concordância interobservador. ESTIMA, Braz. J. Enterostomal Ther., 18: e1720, 2020. https:// doi.org/10.30886/estima.v18.875_PT
\end{abstract}

\begin{abstract}
RESUMO
Objetivo: Verificar a concordância interobservador sobre avaliação de feridas utilizando a ferramenta TIME. Métodos: Estudo de caráter exploratório, não experimental com delineamento transversal e abordagem quantitativa. A população foi constituída por graduandos do oitavo, nono e décimo período do curso de bacharelado em enfermagem, matriculados no segundo semestre do ano de 2018 . Aula expositiva-dialogada sobre a ferramenta TIME foi elaborada e posta aos participantes. Os graduandos foram direcionados ao hospital de ensino para socialização de casos clínicos e avaliação individual de dez lesões de pele com características clínicas diferentes, no paciente hospitalizado utilizando a ferramenta TIME. Para identificar a concordância entre os discentes na utilização da ferramenta TIME, foi utilizado o coeficiente de concordância Kappa. Resultados: Prevaleceu o número de graduandos que estavam cursando o nono período do curso (80\%), sexo feminino (80\%), idade média (23,2 anos). Obteve-se que, considerando a classificação Kappa, houve excelência $(K=1,0)$ na concordância interobservador em todas as etapas da ferramenta TIME. Conclusão: Infere-se que a ferramenta TIME assegura concordância na avaliação de feridas entre graduandos de enfermagem que estão cursando os últimos períodos de curso, podendo contribuir com a melhoria da qualidade do cuidado de enfermagem.
\end{abstract}

DESCRITORES: Avaliação em enfermagem; Ferimentos e lesões; Benchmarking; Estomaterapia; Enfermagem.

\footnotetext{
1. Universidade Estadual do Piauí - Centro de Ciências da Saúde - Teresina/PI -Brasil.

2. Universidade Federal do Piauí - Parnaíba/PI -Brasil.

*Autor correspondente: nflcj@hotmail.com

Recebido: Abr. 18, 2018 | Aceito: Jul. 21, 2020
} 


\begin{abstract}
Objective: To verify interobserver agreement on wound evaluation using the TIME tool. Methods: Exploratory, nonexperimental study with cross-sectional design and quantitative approach. The population consisted of undergraduate students from the eighth, ninth and tenth periods of the baccalaureate nursing course, enrolled in the second semester of 2018. An expositive-dialogued class about the TIME tool was prepared and presented to the participants. The students were directed to the teaching hospital for socialization of clinical cases and individual evaluation of ten skin lesions with different clinical characteristics in the hospitalized patient, using the TIME tool. To identify the agreement between the students, when using the TIME tool, the Kappa agreement coefficient was used. Results: The number of undergraduates who were attending the ninth period of the course (80\%), female (80\%), and average age (23.2 years) prevailed. Considering the Kappa classification, there was excellence $(K=1.0)$ in interobserver agreement in all stages of the TIME tool. Conclusion: It is inferred that the TIME tool ensures agreement in the evaluation of wounds among nursing undergraduates who are attending the last course periods, and may contribute to improving the quality of nursing care.
\end{abstract}

DESCRIPTORS: Nursing assessment; Wounds and injuries; Benchmarking; Enterostomal therapy; Nursing.

\title{
RESUMEN
}

Objetivo: verificar el acuerdo interobservador sobre la evaluación de heridas utilizando la herramienta TIME. Métodos: estudio exploratorio no experimental con diseño transversal y enfoque cuantitativo. La población consistió en estudiantes de pregrado de los períodos octavo, noveno y décimo del curso de enfermería de bachillerato, inscritos en el segundo semestre de 2018. Se preparó y presentó a los participantes una conferencia de diálogo expositivo sobre la herramienta TIME. Los estudiantes fueron dirigidos al hospital universitario para la socialización de casos clínicos y la evaluación individual de diez lesiones cutáneas con diferentes características clínicas en el paciente hospitalizado, utilizando la herramienta TIME. Para identificar el acuerdo entre los estudiantes, utilizando la herramienta TIME, se utilizó el Coeficiente de Acuerdo de Kappa. Resultados: prevaleció el número de estudiantes universitarios que asistían al noveno período del curso (80\%), mujeres (80\%) y edad promedio (23,2 años). Teniendo en cuenta la clasificación de Kappa, hubo excelencia $(K=1.0)$ en el acuerdo interobservador en todas las etapas de la herramienta TIME. Conclusión: se infiere que la herramienta TIME garantiza un acuerdo en la evaluación de heridas entre los estudiantes de pregrado de enfermería que asisten a los últimos períodos del curso y puede contribuir a mejorar la calidad de la atención de enfermería.

DESCRIPTORES: Evaluación en enfermería; Heridas y traumatismos; Benchmarking; Estomaterapia; Enfermería.

\section{INTRODUÇÃO}

O cuidado de enfermagem ao cliente com ferida integra o cotidiano da equipe em todos os níveis de atenção à saúde, na rede pública ou privada, exigindo do profissional enfermeiro conhecimento científico e habilidade diante dos mais diversos aspectos relacionados a prevenção, avaliação e tratamento de uma lesão. Observa-se que esse profissional vem buscando ampliar seus conhecimentos na área do tratamento de feridas ${ }^{1}$.

Assim, o papel do enfermeiro inclui a anamnese e exame físico geral, a avaliação da lesão, a escolha do tratamento e o acompanhamento, além da avaliação dos resultados obtidos com o tratamento estabelecido. Atualmente, existem inúmeras tecnologias e produtos disponíveis para a prevenção e tratamento de feridas. Para determinar a conduta adequada, é necessária uma avaliação fidedigna da ferida, objetivando-se levantar o maior número de fatores que poderão interferir no processo de cicatrização e, consequentemente, na recuperação do cliente. Recentemente, o Conselho Federal de Enfermagem aprovou a Resolução 567/2018, que regulamenta a atuação da equipe de enfermagem no cuidado aos pacientes com feridas, ampliando a atuação desse profissional na área em questão ${ }^{2-4}$.

Apesar disso, estudo realizado em um hospital da Paraíba evidenciou que os enfermeiros apresentam limitações no conhecimento e fragilidades práticas para avaliar as lesões e o paciente, e indicar a cobertura e o tipo de curativo a serem utilizados no controle de sinais e sintomas das lesões ${ }^{5}$.

Dessa forma, considera-se ferida uma ruptura do tegumento, da mucosa ou de qualquer parte do corpo que pode ser causada por um agente químico, biológico ou físico. A ferramenta TIME apresenta como objetivo ser implementada no cuidado às feridas, garantindo a avaliação e permitindo estabelecer as intervenções visando a promoção da cicatrização, considerando os parâmetros avaliados. É um modelo dinâmico formado por quatro parâmetros importantes para o preparo do leito da lesão. Esses parâmetros estão associados aos obstáculos presentes no leito da ferida e que dificultam a cicatrização. $\mathrm{O}$ “T” refere-se à identificação do tecido presente no leito da ferida, o "I" evidencia os sinais de inflamação ou infecção que podem existir, o "M" trata-se da gestão do exsudado e o "E" descreve as características das bordas da ferida $a^{6-8}$. 
Um instrumento de avaliação pode ser considerado simples e de fácil aplicação, contudo o processo para avaliar uma lesão pode ocasionar interpretações divergentes. Uma avaliação errônea pode acarretar sérias consequências, como a determinação de uma conduta inadequada, acarretando complicações e retardando o processo de cicatrização9

Portanto a importância deste estudo justifica-se pela necessidade de se avaliar e discutir o processo de avaliação de feridas, sobretudo acerca da efetividade e da qualidade dessa prática. Considera-se relevante discutir a concordância na avaliação de feridas entre futuros profissionais de enfermagem, pois a inquietação com a qualidade do cuidado deve estar presente já na graduação, essencialmente nos últimos períodos do curso, quando o graduando estará cada vez mais próximo do exercício profissional.

\section{OBJETIVO}

Verificar a concordância interobservador sobre avaliação de feridas utilizando a ferramenta TIME.

\section{MÉTODOS}

Estudo de caráter exploratório, não experimental com delineamento transversal e abordagem quantitativa. Desenvolvido nas dependências de uma instituição de ensino superior (IES), pública e localizada no estado do Piauí, bem como em um hospital geral, público e de ensino, referência na rede do sistema único de saúde em nível de média e altacomplexidade ${ }^{10}$.

A população foi constituída por graduandos do oitavo $(\mathrm{n}=11)$, nono $(\mathrm{n}=20)$ e décimo período $(\mathrm{n}=14)$ do curso de bacharelado em enfermagem da IES, matriculados no segundo semestre do ano de 2018 ( $n=45)$. Foram considerados critérios de inclusão: graduando com idade acima de 18 anos, matriculado regularmente no oitavo, nono ou décimo período do curso de bacharelado em enfermagem, tendo cursado e obtido aprovação nas disciplinas Fundamentos de Enfermagem, Trabalho em Campo IV e Enfermagem em Estomaterapia, ofertadas no quarto e sétimo período do curso,respectivamente, e que aceitaram participar do estudo após esclarecimentos. Excluíram-se participantes que, por algum motivo, ficaram impossibilitados de comparecer e participar de qualquer etapa da coleta de dados. Após aplicação desses critérios, a amostra foi formada por dez participantes, sendo dois discentes que cursavam o oitavo período e oito que cursavam o nono.

Os dados foram coletados no mês de novembro de 2018. Para tanto, os pesquisadores enviaram convite formal via e-mail às turmas, agendando dia e horário para os esclarecimentos, nas dependências da IES. Para aplicação do primeiro instrumento de coleta de dados referente à caracterização da amostra, foi programada data e horário com as turmas envolvidas. Além disso, foi elaborada e posta aos participantes do estudo aula expositiva-dialogada sobre a aplicabilidade da ferramenta TIME e sua dimensão, com duração de duas horas; a aula foi conduzida por uma profissional doutora em enfermagem, com experiência clínica na área de avaliação deferidas.

Depois de realizadas as etapas supracitadas, os graduandos de enfermagem foram direcionados ao hospital de ensino para socialização de casos clínicos e avaliação individual de dez lesões de pele com características clínicas diferentes no paciente hospitalizado, utilizando a ferramenta TIME. Visando a fidedignidade e imparcialidade dos resultados, os casos clínicos foram selecionados por uma profissional doutora em enfermagem e com vasta experiência clínica em avaliação de feridas. O papel da referida profissional foi também o de estabelecer o "padrão ouro" para a avaliação das lesões envolvidas no estudo, utilizando a ferramenta TIME, e ainda o de julgar a avaliação realizada pelos graduandos. Dessa forma, a ferramenta TIME preenchida pelos participantes foi submetida à apreciação, sendo classificada cada dimensão do TIME, em "avaliação incorreta" e "avaliação correta".

Os dados coletados foram digitados no software Microsoft Excel em dupla planilha para validação. Para identificar a concordância entre os discentes na utilização da ferramenta TIME, foi utilizado o coeficiente de concordância Kappa, teste útil para expor a concordância entre dois ou mais juízes em uma avaliação nominal ou ordinal de uma mesma amostra e classificar os respectivos valores em "inconsistente" $(<0)$, "pobre" (0), "ligeira" (0 a 0,20), "considerável” ( 0,21 a $0,40)$, "moderada" (0,41 a 0,60),"substancial" $(0,61$ a 0,80$)$ e "excelente" $(0,81 \text { a } 1)^{11-13 .}$

A pesquisa obedeceu aos aspectos éticos e legais para pesquisa com seres humanos de acordo com as recomendações da Resolução N 466/12 do Conselho Nacional de Saúde do Ministério da Saúde. O estudo foi aprovado pelo Comitê de Ética em Pesquisa com Seres Humanos da Universidade Estadual do Piauí (CEP/UESPI), sob parecer de n $2.903 .039 \mathrm{e}$ Comitê de Ética em Pesquisa com seres humanos do Hospital Getúlio Vargas, sob parecer de n ${ }^{\circ}$ 2.935.595. 


\section{RESULTADOS}

Prevaleceu o número de graduandos que estavam cursando o nono período do curso de enfermagem (80\%), sexo feminino (80\%) e idade média de 23,2 anos.

A Tabela 1 evidencia que houve $100 \%$ de acertos em todas as lesões avaliadas com relação ao tipo de tecido presente no leito da ferida, com exceção da lesão IX, com 80\% para esse item do instrumento. Sobre a primeira e a segunda lesão, a mesma tabela retrata que houve 70 e $60 \%$ de erros quando avaliadas as características do exsudato, respectivamente. Na terceira lesão, houve $60 \%$ de acertos para as características descritas sobre o exsudato e para a avaliação das bordas da referida ferida.

$\mathrm{Na}$ quarta lesão houve $90 \%$ de acertos para a identificação dos sinais de inflamação/infecção, $60 \%$ de acertos para as características do exsudato e para a análise das bordas. A tabela mostra ainda que houve $90 \%$ de erros acerca da avaliação das bordas na quinta ferida. Na sexta lesão houve 100\% de acertos para os sinais de inflamação/infecção e $70 \%$ de erros para descrição do exsudato. Na sétima lesão houve $100 \%$ de acertos acerca dos sinais de inflamação/infecção e 90\% de erros para descrição do exsudato. Já quando avaliada a oitava lesão, houve $100 \%$ de acertos para os sinais de inflamação/ infecção e 60\% de erros quando avaliadas as características do exsudato. Ao ser avaliada a nona lesão, houve $100 \%$ de acertos na identificação dos sinais de inflamação/infecção; já em relação ao tipo e quantidade do exsudato, houve $50 \%$ de erros. Por fim, ao considerar a avaliação da décima lesão, houve $100 \%$ de acertos para a gestão do exsudato; já acerca dos sinais de inflamação/infecção, houve 50\% de erros.

Tabela 1. Avaliação das lesões realizada pelos participantes do estudo, conforme ferramenta TIME e padrão ouro estabelecido por profissional experiente. Teresina, Piauí, Brasil, $2018(n=10)$.

\begin{tabular}{|c|c|c|c|c|c|c|}
\hline \multicolumn{7}{|c|}{ LESÃO I } \\
\hline \multirow{2}{*}{ Variável } & \multicolumn{3}{|c|}{ Acertos } & \multicolumn{3}{|c|}{ Erros } \\
\hline & $\mathrm{n}$ & $\%$ & Média & $\mathrm{n}$ & $\%$ & Média \\
\hline $\mathrm{T}$ & 10 & 100 & 1,0 & 0 & 0 & 0,0 \\
\hline 1 & 7 & 70 & 0,7 & 3 & 30 & 0,3 \\
\hline M & 3 & 30 & 0,3 & 7 & 70 & 0,7 \\
\hline $\mathrm{E}$ & 6 & 60 & 0,6 & 4 & 40 & 0,4 \\
\hline \multicolumn{7}{|c|}{ LESÃO II } \\
\hline \multirow{2}{*}{ Variável } & \multicolumn{3}{|c|}{ Acertos } & \multicolumn{3}{|c|}{ Erros } \\
\hline & $\mathrm{n}$ & $\%$ & Média & $\mathrm{n}$ & $\%$ & Média \\
\hline $\mathrm{T}$ & 10 & 100 & 1,0 & 0 & 0 & 0,0 \\
\hline 1 & 9 & 90 & 0,9 & 1 & 10 & 0,1 \\
\hline M & 4 & 40 & 0,4 & 6 & 60 & 0,6 \\
\hline$E$ & 8 & 80 & 0,8 & 2 & 20 & 0,2 \\
\hline \multicolumn{7}{|c|}{ LESÃO III } \\
\hline \multirow{2}{*}{ Variável } & \multicolumn{3}{|c|}{ Acertos } & \multicolumn{3}{|c|}{ Erros } \\
\hline & $\mathrm{n}$ & $\%$ & Média & $\mathrm{n}$ & $\%$ & Média \\
\hline $\mathrm{T}$ & 10 & 100 & 1,0 & 0 & 0 & 0,0 \\
\hline 1 & 9 & 90 & 0,9 & 1 & 10 & 0,1 \\
\hline M & 6 & 60 & 0,6 & 4 & 40 & 0,4 \\
\hline$E$ & 6 & 60 & 0,6 & 4 & 40 & 0,4 \\
\hline \multicolumn{7}{|c|}{ LESÃO IV } \\
\hline \multirow{2}{*}{ Variável } & \multicolumn{3}{|c|}{ Acertos } & \multicolumn{3}{|c|}{ Erros } \\
\hline & $\mathrm{n}$ & $\%$ & Média & $n$ & $\%$ & Média \\
\hline $\mathrm{T}$ & 10 & 100 & 1,0 & 0 & 0 & 0,0 \\
\hline 1 & 9 & 90 & 0,9 & 1 & 10 & 0,1 \\
\hline M & 6 & 60 & 0,6 & 4 & 40 & 0,4 \\
\hline $\mathrm{E}$ & 6 & 60 & 0,6 & 4 & 40 & 0,4 \\
\hline
\end{tabular}


Tabela 1. Continuação...

\begin{tabular}{|c|c|c|c|c|c|c|}
\hline \multicolumn{7}{|c|}{ LESÃO V } \\
\hline \multirow{2}{*}{ Variável } & \multicolumn{3}{|c|}{ Acertos } & \multicolumn{3}{|c|}{ Erros } \\
\hline & $n$ & $\%$ & Média & $n$ & $\%$ & Média \\
\hline$T$ & 10 & 100 & 1,0 & 0 & 0 & 0,0 \\
\hline 1 & 9 & 90 & 0,9 & 1 & 10 & 0,1 \\
\hline$M$ & 5 & 50 & 0,5 & 5 & 50 & 0,5 \\
\hline $\mathrm{E}$ & 1 & 10 & 0,1 & 9 & 90 & 0,9 \\
\hline \multicolumn{7}{|c|}{ LESÃO VI } \\
\hline \multirow{2}{*}{ Variável } & \multicolumn{3}{|c|}{ Acertos } & \multicolumn{3}{|c|}{ Erros } \\
\hline & $\mathrm{n}$ & $\%$ & Média & $\mathrm{n}$ & $\%$ & Média \\
\hline$T$ & 10 & 100 & 1,0 & 0 & 0 & 0,0 \\
\hline 1 & 10 & 100 & 1,0 & 0 & 0 & 0,0 \\
\hline$M$ & 3 & 30 & 0,3 & 7 & 70 & 0,7 \\
\hline$E$ & 6 & 60 & 0,6 & 4 & 40 & 0,4 \\
\hline \multicolumn{7}{|c|}{ LESÃO VII } \\
\hline \multirow{2}{*}{ Variável } & \multicolumn{3}{|c|}{ Acertos } & \multicolumn{3}{|c|}{ Erros } \\
\hline & $\mathrm{n}$ & $\%$ & Média & $\mathrm{n}$ & $\%$ & Média \\
\hline $\mathrm{T}$ & 10 & 100 & 1,0 & 0 & 0 & 0,0 \\
\hline 1 & 10 & 100 & 1,0 & 0 & 0 & 0,0 \\
\hline$M$ & 1 & 10 & 0,1 & 9 & 90 & 0,9 \\
\hline $\mathrm{E}$ & 7 & 70 & 0,7 & 3 & 30 & 0,3 \\
\hline \multicolumn{7}{|c|}{ LESÃO VIII } \\
\hline \multirow{2}{*}{ Variável } & \multicolumn{3}{|c|}{ Acertos } & \multicolumn{3}{|c|}{ Erros } \\
\hline & $\mathrm{n}$ & $\%$ & Média & $n$ & $\%$ & Média \\
\hline$T$ & 10 & 100 & 1,0 & 0 & 0 & 0,0 \\
\hline 1 & 10 & 100 & 1,0 & 0 & 0 & 0,0 \\
\hline M & 4 & 40 & 0,4 & 6 & 60 & 0,6 \\
\hline$E$ & 5 & 50 & 0,5 & 5 & 50 & 0,5 \\
\hline \multicolumn{7}{|c|}{ LESÃO IX } \\
\hline \multirow{2}{*}{ Variável } & \multicolumn{3}{|c|}{ Acertos } & \multicolumn{3}{|c|}{ Erros } \\
\hline & $\mathrm{n}$ & $\%$ & Média & $n$ & $\%$ & Média \\
\hline $\mathrm{T}$ & 8 & 80 & 0,8 & 2 & 20 & 0,2 \\
\hline I & 10 & 100 & 1,0 & 0 & 0 & 0,0 \\
\hline M & 5 & 50 & 0,5 & 5 & 50 & 0,5 \\
\hline$E$ & 8 & 80 & 0.8 & 2 & 20 & 0,2 \\
\hline \multicolumn{7}{|c|}{ LESÃO X } \\
\hline \multirow{2}{*}{ Variável } & \multicolumn{3}{|c|}{ Acertos } & \multicolumn{3}{|c|}{ Erros } \\
\hline & $n$ & $\%$ & Média & $\mathrm{n}$ & $\%$ & Média \\
\hline $\mathrm{T}$ & 10 & 100 & 1,0 & 0 & 0 & 0,0 \\
\hline 1 & 5 & 50 & 0,5 & 5 & 50 & 0,5 \\
\hline$M$ & 10 & 100 & 1,0 & 0 & 0 & 0,0 \\
\hline$E$ & 7 & 70 & 0,7 & 3 & 30 & 0,3 \\
\hline
\end{tabular}

Fonte: Pesquisa direta. 
Na Tabela 2 evidencia-se o coeficiente de concordância Kappa, conforme avaliação realizada pelos participantes nas dez lesões que foram objeto deste estudo, utilizando a ferramenta TIME e considerando o padrão ouro previamente estabelecido. Obteve-se que, considerando a classificação Kappa, houve excelência $(K=1,0)$ na concordância interobservador em todas as etapas da ferramenta TIME.

Tabela 2. Concordância geral entre os participantes do estudo na avaliação das lesões, conforme Coeficiente Kappa. Teresina, Piauí, Brasil, $2018(\mathrm{n}=10)$.

\begin{tabular}{|c|c|c|}
\hline \multirow{2}{*}{ Variável } & \multicolumn{2}{|c|}{ Concordância } \\
\hline & $\mathrm{K}^{*}$ & Classificação \\
\hline $\mathrm{T}$ & 1,00 & Excelente \\
\hline 1 & 1,00 & Excelente \\
\hline $\mathrm{M}$ & 1,00 & Excelente \\
\hline E & 1,00 & Excelente \\
\hline
\end{tabular}

*Coeficiente de concordância Kappa.

Fonte: Pesquisa direta.

\section{DISCUSSÃO}

Prevaleceram neste estudo os participantes que cursavam o nono período do curso de graduação em enfermagem.Esse resultado está associado à dificuldade encontrada para ajustar horários iguais para todos os participantes durante as etapas de coleta de dados, haja vista as limitações relacionadas aos compromissos acadêmicos, uma vez que o referido curso funciona em tempo integral.

Destacou-se o sexo feminino com um percentual considerável, o que corrobora as características da profissão almejada pelos participantes. Estudo transversal realizado com um universo de 1,8 milhão de profissionais de enfermagem, cujo objetivo era o de traçar o perfil da equipe de enfermagem no Brasil, demonstrou que, no país inteiro, 86,2\% (357.551) dos profissionais enfermeiros pertencem ao sexo feminino. No Piauí, o número fica em 84,6\% (5.794) ${ }^{14}$.

Acerca do tipo de tecido presente no leito da ferida, o estudo evidenciou que houve excelência $(K=1,0)$ na concordância interobservador, o que vai ao encontro de um estudo realizado em ambulatórios de cinco hospitais da grande São Paulo, junto a pacientes com úlceras crônicas de perna e que teve como objetivo testar a confiabilidade entre enfermeiras clínicas e estomaterapeutas sobre o pressure ulcer scale for healing (PUSH), em sua versão adaptada para a língua portuguesa. A pesquisa mostrou que houve concordância total $(K=1,0)$ entre as respostas de todas as observadoras com relação à avaliação da aparência do leito da ferida. Em adição, é importante ressaltar que os resultados deste estudo mostram que, além da concordância excelente, os participantes obtiveram de 80 a $100 \%$ de acertos nesse item da ferramentaTIME ${ }^{15}$.

Acerca dos sinais de infecção/inflamação avaliados, Kappa apresentou excelência $(K=1,0)$. No entanto, foi encontrado na literatura um estudo realizado com discentes do último período do curso de graduação em enfermagem em uma instituição pública do Paraná que evidenciou uma carência na identificação de sinais de inflamação/infecção e de entendimento sobre a diferença entre contaminação e colonização de feridas. No mesmo estudo, acerca das peculiaridades de uma lesão com sinais de inflamação/infecção, a maior parte dos acadêmicos não citou hipertermia (96\%), edema (70\%), calor local (66,7\%) e dor $(60 \%)^{16}$. De fato, embora os participantes deste estudo tenham obtido concordância excelente, o percentual de acertos para esse item variou, na maior parte das lesões, entre $80 \mathrm{a}$ $100 \%$, obtendo apenas $50 \%$ em uma das lesões.

Pesquisa descritiva, exploratória, com análise quantitativa, realizada no Hospital de Base de Ribeirão Preto, Brasil, que teve como objetivo avaliar a concordância entre os enfermeiros assistenciais quanto à avaliação e à classificação do risco para lesão por pressão em pacientes internados em unidades de terapia intensiva, mostrou que a subescala "umidade" foi a que obteve menor concordância entre os enfermeiros e a pesquisadora, classificada como pobre $(\mathrm{K}=0,0)$ em duas unidades de terapia intensiva e nenhum grau de concordância na terceira unidade $(K=-0,04)^{17}$.

Apesar de a pesquisa citada acima ter objeto de estudo distinto da presente investigação, esses resultados apontam a 
subjetividade que envolve as avaliações do item "umidade" por parte do enfermeiro. O fato chama a atenção para a provável necessidade de maior ênfase nesse ponto, em específico durante a formação e o aperfeiçoamento desses profissionais.

Além disso, estudiosos do assunto enfatizam que o exsudato é componente essencial a ser observado e controlado para a cicatrização e que, além do volume, há de se considerar o aspecto e a composição, pois há evidências de que a composição do fluido da ferida crônica é tão importante quanto a sua quantidade. Em comparação com a ferida aguda, foi demonstrado que o exsudato da ferida crônica inibe o crescimento de fibroblastos, necessários para a deposição e a organização do colágeno, e que este tem níveis aumentados de citocinas pró-inflamatórias, radicais livres de oxigênio e proteases, prolongando o estágio inflamatório da cicatrização da ferida. Concordando com essas observações, o percentual de acerto dos participantes deste estudo para o item "M" da ferramenta TIME, variou de 10 a $100 \%$ nas lesões avaliadas ${ }^{18}$.

Ao serem avaliadas as propriedades das bordas das lesões, o Kappa apresentou concordância excelente $(K=1,0)$. Não foram encontradas evidências científicas na literatura acerca da concordância ou discordância no item "bordas".

Vale ressaltar que, na instituição de ensino estudada, a disciplina Enfermagem em Estomaterapia compõe a grade curricular obrigatória do curso de graduação em Enfermagem desde 2017, sendo ofertada no sétimo período, com carga horária de 45 horas por semestre. Sabendo da relevância do conhecimento adquirido na disciplina, compreende-se que os resultados obtidos neste estudo estão diretamente associados a essa formação.

Foram encontradas limitações para realização da pesquisa, relacionadas à dificuldade em conciliar horários e dias para a população estar presente nos momentos de coleta de dados, o que prejudicou o tamanho da amostra, a carência de estudos na literatura que abordem o processo de avaliação de feridas, principalmente sobre o uso da ferramenta TIME, e a concordância interobservador, sendo encontrados com prevalência artigos abordando apenas a temática de tratamento deferidas.
Portanto considera-se relevante a continuação da discussão sobre o processo de avaliação de feridas, sobretudo acerca da efetividade e da qualidade dessa prática presente na rotina do profissional enfermeiro. Dessa forma, novos estudos deverão ser realizados objetivando ampliar a amostra de participantes e aumentar o número de feridas a serem avaliadas, bem como incluir não só discentes de graduação, mas também profissionais e especialistas na área em questão.

\section{CONCLUSÃO}

O estudo pôde, por meio da metodologia utilizada, responder ao seu objetivo de forma satisfatória. A concordância interobservador sobre avaliação de feridas, questão dessa pesquisa, mostrou-se "excelente", haja vista os valores de Kappa $(K=1,0)$ atribuídos a cada dimensão da ferramenta TIME.

Portanto, apesar de os percentuais de acertos deste estudo demonstrarem ainda algumas dificuldades no processo de avaliação de feridas, por parte dos participantes, infere-se que a ferramenta TIME assegura concordância na avaliação de feridas, entre graduandos de enfermagem que estão cursando os últimos períodos de curso, podendo contribuir com a melhoria da qualidade do cuidado de enfermagem.

\section{CONTRIBUIÇÃO DOS AUTORES}

Conceitualização, Rocha ESB; Metodologia, Rocha ESB; Investigação, Coutinho Júnior NFL; Ferreira LFO e Rocha ESB; Análise Formal, Carvalho MRD; Bezerra SMG e Branco NFLC; Curadoria de Dados, Rocha Júnior K; Redação Primeira versão, Rocha ESB e CoutinhoJúnior NFL; RedaçãoRevisão \& Edição, Rocha ESB; Coutinho Júnior NFL; Bezerra SMG e Branco NFLC; Supervisão, Carvalho MRD e Rocha ESB; Administração do Projeto, Rocha ESB.

\section{REFERÊNCIAS}

1. Campos MGCA, Sousa ATO, Vasconcelos JMB, Lucena SAP, Gomes SKA. Feridas complexas e estomias: aspectos preventivos e manejo clínico. João Pessoa: Ideia;2016.

2. Oliveira FP, Oliveira BGRB, Santana RF, Silva BP, Candido JSC. Classificações de intervenções e resultados de enfermagem em pacientes com feridas: mapeamento cruzado. Rev Gaúcha Enferm. 2016;37(2):e55033. https:// doi.org/10.1590/1983-1447.2016.02.55033

3. Squizatto RH, Braz RM, Lopes AO, Rafaldini BP, Almeida DB, Poletti NAA. Perfil dos usuários atendidos em ambulatório 
de cuidado com feridas. Cogitare Enferm. 2017;22(1):1-9. https://doi.org/10.5380/ce.v22i1.48472

4. Brasil. Conselho Federal de Enfermagem (COFEN). Resolução COFEN n.567/2018 de 7 de fevereiro de 2018. Regulamenta a atuação da equipe de enfermagem no cuidado aos pacientes com feridas. 2018. [citado em 8 Abr 2018]. Disponível em: http://www.cofen.gov.br/resolucaocofen-no-567-2018_60340.html

5. Agra G, Medeiros MVS, Brito DTF, Sousa ATO, Formiga NS, Costa MML. Conhecimento e prática de enfermeiros no cuidado a pacientes com feridas tumorais malignas. Rev Cuid. 2017;8(3):1849-62. https://doi.org/10.15649/cuidarte. v8i3.441

6. Aron S, Gamba MA. Preparo do leito da ferida e a história do TIME. ESTIMA, Braz J Enterostomal Ther. 2009;7(4):1.

7. Rocha DM, Bezerra SMG, Santos RR, Moreira L. Avaliação do leito da ferida e utilização da ferramenta TIME. In: Bezerra SMG, Rocha DM, Nogueira LT, organizadores. Protocolo de prevenção, avaliação e tratamento de lesões de pele do Município de Teresina. Teresina: EDUESPI; 2016. p.19-23.

8. Machado FS, Costa AEK, Pissaia LF, Beschorner CE, Moreschi C. Perspective of the nurse in front of nursing care in the treatment of wounds in hospitals. R Epidemiol Control Infec. 2017;7(3):134-9. https://doi.org/10.17058/reci.v7i3.8920

9. Bajay HM, Araújo IEM. Validação e confiabilidade de um instrumento de avaliação de feridas. Acta Paul Enferm.2006;19(3):290-5. https://doi.org/10.1590/S010321002006000300006
10. Brasil. Secretária de Estado da Saúde do Piauí. Hospital Getúlio Vargas: Referência para o Meio Norte do Brasil. Quem somos. 2018. [citado em 8 Abr 2018]. Disponível em: http://www.hgv.pi.gov.br/quem.php

11. Martins GA. Estatística geral e aplicada. Rio de Janeiro:Atlas; 2009.

12. Triola MF. Introdução à estatística. Rio de Janeiro: LTC; 2008.

13. Landis JR, Kock GG. The measurement of observer agreement for categorical data. Biometrics, 1977;33(1):15974. https://doi.org/10.2307/2529310

14. Conselho Federal de Enfermagem (COFEN). Pesquisa inédita traça perfil da enfermagem. 2018. [citado em 5 Dez 2018]. Disponível em: http://www.cofen.gov.br/pesquisa-ineditatraca-perfil-da-enfermagem_31258.html

15. Santos VLCG, Sellmer D, Massulo MME. Inter rater reliability of Pressure Ulcer Scale for Healing (PUSH) in patients with chronic leg ulcers.Rev Latino-Am Enfermagem. 2007;15(3):391-6. https://doi.org/10.1590/S0104-11692007000300005

16. Baratieri $T$, Sangaleti $C T$, Trincaus MR. Conhecimento de acadêmicos de enfermagem sobre avaliação e tratamento de feridas. Rev Enferm Atenção Saúde.2015;4(1):2-15.

17. Simão CMF, Caliri MHL, Santos CB. Concordância entre enfermeiros quanto ao risco dos pacientes para úlcera por pressão. Acta Paul Enferm. 2013;26(1):30-5. https://doi. org/10.1590/S0103-21002013000100006

18. Harries RL, Bosanquet DC, Harding KG. Wound bed preparation: TIME for an update. Int Wound J. 2016;13(S3):814. https://doi.org/10.1111/iwj.12662 